\title{
Formulation and Evaluation of Nisoldipine Loaded Solid Lipid Nanoparticles and Nanostructured Lipid Carriers: Application to Transdermal Delivery
}

\author{
Suddala Shirisha ${ }^{1}$, Ankam Saraswathi ${ }^{2}$, Sunit Kumar Sahoo ${ }^{1, *}$, Yamsani Madhusudan Rao³ \\ ${ }^{1}$ Department of Pharmaceutical Sciences, Utkal University, Vani Vihar, Bhubaneswar, Odisha, INDIA. \\ ${ }^{2}$ University College of Pharmaceutical Sciences, Kakatiya University, Warangal, INDIA. \\ ${ }^{3}$ Vaagdevi Group of Pharmacy Colleges, Warangal, Telangana, INDIA.
}

\begin{abstract}
Background: Nisoldipine is an anti hypertensive drug. Nisoldipine exhibits poor oral bioavailability $(5 \%)$ because of rapid metabolism in the gut and liver. To overcome hepatic first pass metabolism and to enhance bioavailability, lipid based drug delivery systems (Solid lipid nanoparticles (SLN) and nano structure lipid carriers (NLC)) can be exploited. Objectives: In this work, effort was made to prepare novel particulate carrier systems such as stable Solid Lipid Nanoparticles and Nanostructured Lipid Carriers for transdermal delivery of Nisoldipine (NSP). For this investigation, Carbopol 934 used as gel forming agent for hydrogel preparation. Methods: Aqueous dispersions of lipid nanoparticles made from Dynasan 114, 116, 118 were prepared by hot homogenization technique followed by ultrasonication and then optimized formulations of SLN and NLCs were incorporated into the freshly prepared hydrogels. Prepared lipid nanoparticles were characterized for particle size, zeta potential, entrapment efficiency, stability and in-vitro release profile. Also percutaneous permeation of SLN and NLCs were investigated in rat abdominal skin. Results: Analyzing the particles size by photon correlation spectroscopy (PCS) using Malven zeta sizer, which shows that the SLN and NLCs were in the range of $130-330 \mathrm{~nm}$ at room temperature. For all the tested formulations (SLN and NLCs), the entrapment efficiency was $72-97 \%$. In-vitro drug release studies were performed for $24 \mathrm{hr}$. In these two cases the percentage drug release from gels enriched with SLN/NLC showed sustained release over period of $24 \mathrm{hr}$. In agreement with these results NSPNLC (Nisoldipine nanostructured lipid carriers) dispersion showed faster release. Formulation E4 showed faster release has less particle size and more zeta potential. Conclusion: Based on these, it was selected for further stability and ex-vivo studies. Both the SLN and NLC showed a sustained drug release over a period of $24 \mathrm{hr}$, but the sustained effect was more pronounced with the SLN and NLC gel formulations.
\end{abstract}

Key words: Nisoldipine, Solid lipid nanoparticles, Nanostructured lipid carriers, In-vitro release studies, Ex-vivo permeation studies, Lyophilization.

\section{INTRODUCTION}

The development of a novel drug delivery system for a particular drug is difficult due to the differences of the drug solubility in the vehicle components and the vast range in cutaneous fluxes. In the majority of pharmaceutical formulations intended for transdermal/topical and dermatological therapy the drug molecules are dissolved in a liquid phase of oil in water $(\mathrm{o} / \mathrm{w})$ or water in oil (w/o) emulsion. However, due to the low viscosity of the inner phase of the systems it is difficult to achieve a prolonged or controlled release of a drug. Noninvasive drug delivery systems are gaining market share at the expense of oral and parenteral delivery. Technology advances are expanding the number of drug candidates that can be solubilized and delivered via transdermal gels. The trend towards increased home care and
Submission Date: 16-09-2019; Revision Date: 15-11-2019; Accepted Date: 12-05-2020

DOI: 10.5530/ijper.54.2s.68 Correspondence: Dr. Sunit Kumar Sahoo Lecturer, University Department of Pharmaceutical Sciences, Utkal University, Vani Vihar, Bhubaneswar-751004, Odisha, INDIA.

Phone: +91-9437134184

E-mail: sahoosunitkumar@ gmail.com

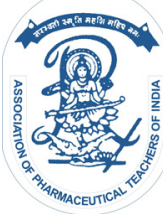

www.ijper.org 
drug therapy, self-administration will accelerate interest in transdermal gel formulations. ${ }^{1}$

In order to overcome the drawbacks associated with the colloidal systems, such as emulsions, liposomes and polymeric nanoparticles, Solid lipid nanoparticles were developed at the beginning of the nineties. ${ }^{2}$ SLN having a mean particle size in submicron size between $50 \mathrm{~nm}$ and $1000 \mathrm{~nm}$ and are made up of materials which are biocompatible and biodegradable with capability of incorporating hydrophilic and liphophilic drugs. ${ }^{3}$ A clear advantage of the use of lipid particles as drug carrier systems is that the matrix is composed of physiological components, i.e. excipients with generally recognized as safe (GRAS) status for oral and topical administration, which decreases the danger of acute and chronic toxicity. Preparation of SLN by exchanging the liquid lipid (oil) of the emulsions by a solid lipid, ${ }^{4}$ which can bring many advantages in comparison to liquid core. ${ }^{5}$ SLNs are low cost products. In fact, the excipients and production lines are relatively cheap.

The second generation of lipid nanoparticles are called nanostructure lipid carriers (NLC). ${ }^{67}$ The difference between NLC and SLN is the fact that the concept of these former is performed by nano structuring the lipid matrix, in order to increase the drug loading and to prevent its leakage, giving more flexibility for modulation of drug loading and to prevent its leakage, giving more flexibility for modulation of drug release. For achieving of this approach by mixing solid lipid with liquid lipids in NLC, instead of highly purified lipids with relatively similar molecules in SLN. This mixture has to be solid at least at $40^{\circ} \mathrm{C}$. The result is less ordered lipid matrix with many imperfections, which can accommodate a higher amount of drug. ${ }^{7,8}$ Different substances have been entrapped into lipid nanoparticles, ranging from lipophilic and hydrophilic molecules, including labile compounds, such as peptides and proteins during the last 10 years. ${ }^{9}$ The purpose of this project is to prepare and characterize solid lipid nanoparticles (SLN) and nanostructured lipid carriers (NLC) loaded with Nisoldipine, for transdermal drug delivery. The possibilities to use lipid nanoparticles for sustained or controlled release application have been discussed.

Nisoldipine is an anti hypertensive drug. Nisoldipine exhibits poor oral bioavailability (5\%) because of rapid metabolism in the gut and liver. To overcome hepatic first pass metabolism and to enhance bioavailability, lipid based drug delivery systems (SLN and NLC) can be exploited. These systems incorporated into hydrogels for transdermal application increase the bioavailability. In the present study solid lipid nanoparticles and nano structured lipid carriers are employed to incorporate
Nisoldipine to bypass the first pass metabolism, thereby to increase its bioavailability and to improve activity.

\section{MATERIALS}

Nisoldipine obtained as a gift sample from Aurabindo Pharma, Hyderabad, India. Dynasan 114, 116 were generously supplied by Sasol, Witten, Germany. Dynasan 118 and Oleic acid were supplied from S.D. Fine-Chem Ltd, India. Carbopal 934p, Glycerol and Dialysis membrane-70 were purchased from Hi-media (Mumbai, India). Soya Lecithin (Lipoid E80) was obtained from Lipoid, Germany. Centrisart filters (molecular weight cutoff 20,000) were purchased from Sartorius (Goettingen, Germany). The other chemicals were of analytical reagent grade.

\section{METHODS}

\section{Characterization by Differential Scanning Calorimetry (DSC)}

DSC is one of the basic techniques used to investigate drug-excipients compatibility. It quantifies the enthalphic changes during endothermic or exothermic effects. The instrument was calibrated with indium (calibration standard, purity $>99.99 \%$ ) for melting point and heat of fusion. About $\sim 5 \mathrm{mg}$ sample was taken for analysis into standard aluminum pans. An empty pan was used as reference. The heating rate was increased at the rate of $20^{\circ} \mathrm{C} / \mathrm{min}$ with heating range of $20-200^{\circ} \mathrm{C}$ and the obtained thermo grams were observed for any type of interaction. DSC analysis of Nisoldipine (NSP), Dynasan-114, 116 and 118 and lyophilized Nisoldipine products were analyzed.

\section{Preparation of Aqueous SLN and NLCs Dispersions and Gels of Nisoldipine}

Nisoldipine (drug $10 \mathrm{mg}$ ), solid lipid (100-300mg) and soya lecithin $(100 \mathrm{mg}$ ) were dissolved in $10 \mathrm{~mL}$ mixture of chloroform and methanol (1:1). Organic solvents were completely removed using a Rota evaporator (Laborota 4000, Heidoph, Germany). A drug embedded lipid layer was melted by heating at $58^{\circ} \mathrm{C}$ above melting point of the lipid. Aqueous phase was prepared by dissolving polaxamer $407(0.5$ to $2 \% \mathrm{w} / \mathrm{v})$ in double distilled water (sufficient to produce $10 \mathrm{~mL}$ of preparation) and heated to the same temperature of the oil phase. Hot aqueous phase was added to the oil phase and homogenization was carried out (at 12,000 rpm) using a Diax 900 homogenizer (Heidolph, Germany) for 10min. Coarse hot oil in water emulsion so obtained was ultrasonicated (12T-probe) using Vibracell probe 
sonicator (Bandelin, Germany) for 20 min. Nisoldipine loaded solid lipid nanoparticles were obtained by allowing hot nano emulsion to cool to room temperature. ${ }^{10}$ Nanostructured Lipid Carriers (NLC) was prepared in exactly the same manner as the SLN dispersions; only replacing 30\% of the solid lipid matrix by oleic acid. Quantity of ingredients used for the preparation of SLNs and NLCs of Nisoldipine given in the Table 1.

\section{Optimization of Formulation variables}

For development of SLN/NLCs optimize the formulation variables like lipid content, concentration of surfactant and optimize the process variables like homogenization time, ultrasonication time was observed. For effect of lipid content three different amounts of lipid (100, 200 and 300) were selected for the preparation of SLN of Nisoldipine. The formulations were stored at room temperature and examined for physical stability. The formulations were also used for in-vitro release studies. In order to observe the effect of polaxamer 407 concentration on particle size and physical stability, different concentrations of polaxamer $407(0.5 \%, 1.75 \%$ and $2 \%)$ were used in the formulation of SLN and NLCs of Nisoldipine. The homogenization time was varied from $5 \mathrm{~min}$ to $10 \mathrm{~min}$ while homogenization speed (12,000rpm) and ultra sonication time were kept constant. In another study, the homogenization time was kept constant at $10 \mathrm{~min}$ and the ultrasonication time was varied from 15 to $20 \mathrm{~min}$.

\section{Preparation of gels enriched with Lipid Nanoparticles}

Gels were prepared using Carbopol 934p (2\%) polymer. For the preparation of hydrogel, the gel forming polymer (was dispersed in double distilled water containing glycerol (10\%). $20 \%$ of aqueous SLN and NLCs dispersions and hydrogels were mixed in a high-speed stirrer (Remi, Mumbai, India) at approximately $100 \mathrm{rpm}$ for 5 min to yield hydrogels containing a final concentration of lipid nanoparticles about $5 \%{ }^{11}$ The hydrogels composed of carbopol 934p were adjusted to $\mathrm{pH} 6.5$ with triethanolamine. The dispersions of SLN and NLCs were used as reference.

\section{Evaluation of SLN and NLCs dispersion and Gels Physicochemical Properties}

After centrifugation the dispersions of SLN and NLCs were characterized for their physicochemical properties such as color, odor and stability. The gels enriched with SLN and NLCs were evaluated for color, odor and $\mathrm{pH}$. Measurement of Particle size, PDI and zeta potential of SLN.
The size, PDI and zetapotential of Nisoldipine SLNs and NLCs were measured using photon correlation spectroscopy (PCS), with the help of a Malvern Zetasizer (Nano ZS90). The prepared SLN and NLCs were diluted appropriately with the aqueous phase of the formulation for the measurements and the $\mathrm{pH}$ of diluted samples ranged from 6.8 to 7.4. Measurement of zeta potential was done at $25^{\circ} \mathrm{C}$ and the strength of electric field strength was around $23.2 \mathrm{~V} / \mathrm{cm}$.

\section{Transmission Electron Microscopy}

Transmission electron microscope (TEM) images were obtained by using a Jeol 3010, Japan, transmission electron microscope operated at $300 \mathrm{keV}$. TEM samples were prepared by dropping dispersion of the particles on copper grid supported formvar films.

\section{Determination of drug content and entrapment efficiency}

About $0.2 \mathrm{~mL}$ of the NSPSLN/NSPNLCs formulation was diluted to $10 \mathrm{~mL}$ with chloroform and methanol mixture (1:1) and then further dilutions were made with the mixture of above solvents. The diluted samples were estimated by UV- visible spectrophotometer at $\lambda_{\max }$ $238 \mathrm{~nm}$ for the amount of Nisoldipine present.

Entrapment efficiency of the system was determined by measuring the concentration of free drug (unentrapped) in aqueous medium as reported previously by Venkateshwarlu and Manjunath, 2004. ${ }^{12}$ The aqueous medium was separated by ultracentrifugation using centrisart tubes (Sartorius, USA) which consist of filter membrane (M.Wt.cut off 20,000 Da) at the base of the sample recovery chamber. About $1 \mathrm{~mL}$ of the undiluted sample of NSPSLN/NSPNLCs formulation was placed in the outer chamber and sample recovery chamber placed on top of the sample and centrifuged at 12,000 rpm for 15 min. The SLN/NLCs along with the encapsulated drug remain in the outer chamber and aqueous phase moves into the sample recovery chamber through the filter membrane. The amount of the NSP in the aqueous phase was estimated by UV-spectrophotometer at $\lambda_{\max } 238 \mathrm{~nm}$. The entrapment efficiency of Nisoldipine SLN/NLC was calculated using the formula:

Entrapment efficiency $=\frac{\text { Amount of Nisoldipine in lipid phase }}{\text { Assay value }} \times 100$

\section{In vitro drug release studies of SLN and NLC and gels enriched with SLN and NLC}

In-vitro release studies were performed using modified franz diffusion cells. ${ }^{13}$ Dialysis membrane having pore size $2.4 \mathrm{~nm}$ and molecular weight cut off between $12,000-14,000$ was used for the release studies. Dialysis membrane was soaked overnight in double distilled 
water for $12 \mathrm{~h}$ prior mounting in a modified franz diffusion cell. $3 \mathrm{~mL}$ of SLN/NLCs dispersion/ 0.5g of lipid nanoparticles incorporated hydrogel were placed in the donor compartment and the phosphate buffer $\mathrm{pH} 7.4$ with $0.75 \%$ sodium lauryl sulphate (SLS) was filled into receptor compartment. During the process, the receptor side solution was maintained at $37 \pm 0.5^{\circ} \mathrm{C}$ and stirred at $800 \mathrm{rpm}$ with Teflon coated magnetic stirring beads. At fixed time intervals, $1 \mathrm{~mL}$ of sample was withdrawn from receptor compartment through side tube analyzed by UV- visible spectrophotometer at $\lambda_{\max } 238 \mathrm{~nm}$.

Data obtained from in-vitro release studies were fitted to various kinetic equations to find out the mechanism of Nisoldipine release from NSPSLN, NSPNLC, NSPSLN gel and NSPNLC gel. The kinetic models used were zero order equation, first order equation, higuchi release and korsemayer peppas equation.

\section{Ex vivo permeation studies}

The ex-vivo permeation studies for optimized formulations was performed in sodium dihydrogen phosphate buffer (7.4) with $0.75 \%$ SLS using Franz's diffusion cell with diffusional area of $3.14 \mathrm{~cm}^{2}$. In these studies, rats abdominal skin is used for the permeation. ${ }^{14}$ The excised rat skin was set in place with the stratum corneum facing the donor compartment and the dermis facing the receptor. $3 \mathrm{~mL}$ of SLN/NLC dispersion of Nisoldipine and gels enriched with SLN/NLC sample was placed on the skin surface in the donor compartment and $26 \mathrm{~mL}$ of media was placed in the receptor compartment. During the experiments, the solution in receptor side was maintained at $37 \pm 0.5^{\circ} \mathrm{C}$ and stirred at $800 \mathrm{rpm}$ with Teflon coated magnetic beads. After application of the test formulation on the donor side $1 \mathrm{~mL}$ of samples were collected from a receptor compartment at designated time intervals $(1,2,4,8,12,20$ and $24 \mathrm{hr}$ ). Thereafter, an equivalent volume of receptor fluid was supplied to the receiver compartment immediately after each sample collection. The amount of NSP in receptor fluids were analyzed by UV-Visible spectrophotometer at $\lambda_{\max } 238 \mathrm{~nm}^{15}$

Nisoldipine fluxes through the skin were calculated by plotting the cumulative amount of drug permeated through skin against time and determining the slope of the linear portion of the curve. At steady state, drug fluxes $\left(\mu \mathrm{g} / \mathrm{cm}^{2} / \mathrm{hr}\right)$ were calculated by dividing slope of the linear portion of the curve by the area of the skin surface through which diffusion took place. The target flux is calculated using the following formula. ${ }^{16}$

$$
\mathrm{J}_{\text {target }}=\mathrm{C}_{\mathrm{SS}} \mathrm{CL}_{\mathrm{T}} \mathrm{BW} / \mathrm{A}
$$

Where, $\mathrm{A}$ is the effective diffusional area $\left(3.14 \mathrm{~cm}^{2}\right)$, $\mathrm{BW}$ the standard human body weight of $60 \mathrm{~kg}, \mathrm{C}_{\mathrm{SS}}$ the Nisoldipine concentration at the therapeutic level and $\mathrm{CL}_{\mathrm{T}}$ the total clearance.

\section{Physical stability studies}

Nisoldipine loaded solid lipid nanoparticles were stored at room temperature and refrigerated temperature for 60 days and average size, zeta potential and poly dispersity index were determined. The number of samples estimated was in triplicate $(n=3)$.

\section{Lyophilisation of NLCs preparation}

Lyophilisation was done to enhance the stability of the NLCs preparation. To the NLCs preparation 10\% mannitol was used as a cryoprotectant. The samples were frozen overnight and those frozen samples were subjected to lyophilisation at $-56^{\circ} \mathrm{C}$ for a period of $24 \mathrm{hr}$.

\section{RESULTS AND DISCUSSION}

\section{Characterization of the investigated formulation}

NSPSLN were prepared by hot homogenization followed by ultrasonication method using three different solid lipids like Dynasan 114, 116 and 118. For optimizing the process variables, optimal particle size and PDI were observed when the homogenization was carried out for a period of $10 \mathrm{~min}$ and ultrasonication time was carried out for a period of $20 \mathrm{~min}$. Further increase in homogenization time and ultrasonication time had no considerable effect on particle size. Similarly, for selecting formulation variables, optimized lipid concentration was $200 \mathrm{mg}$ increasing the lipid concentration beyond $300 \mathrm{mg}$ resulted higher particle size and release was slowed down. The optimized concentration of Soya lecithin and surfactant (poloxamer 407) was found to be $100 \mathrm{mg}$ and $1.75 \% \mathrm{w} / \mathrm{v}$ respectively, the final composition of NSPSLN and NSPNLCs were shown in Table 1.

The optimized SLN formulations with three different lipids (Dynasan 114, 116, 118) are F4, S4 and E4. The best of these three formulations i.e., E4 is used to prepare the NLCs. NLCs were prepared in exactly the same manner as the SLN dispersions, only partially replacing $30 \%$ of the solid lipid matrix by liquid lipid (oleic acid), which was discussed earlier.

For this investigation, hydrogel was prepared using optimal stabilizer combination of water, gel forming agent (Carbopol 934) and hydrating agent is glycerol. The selected aqueous dispersions of NSPSLN and NSPNLCs were admixed to the freshly prepared hydrogels. 


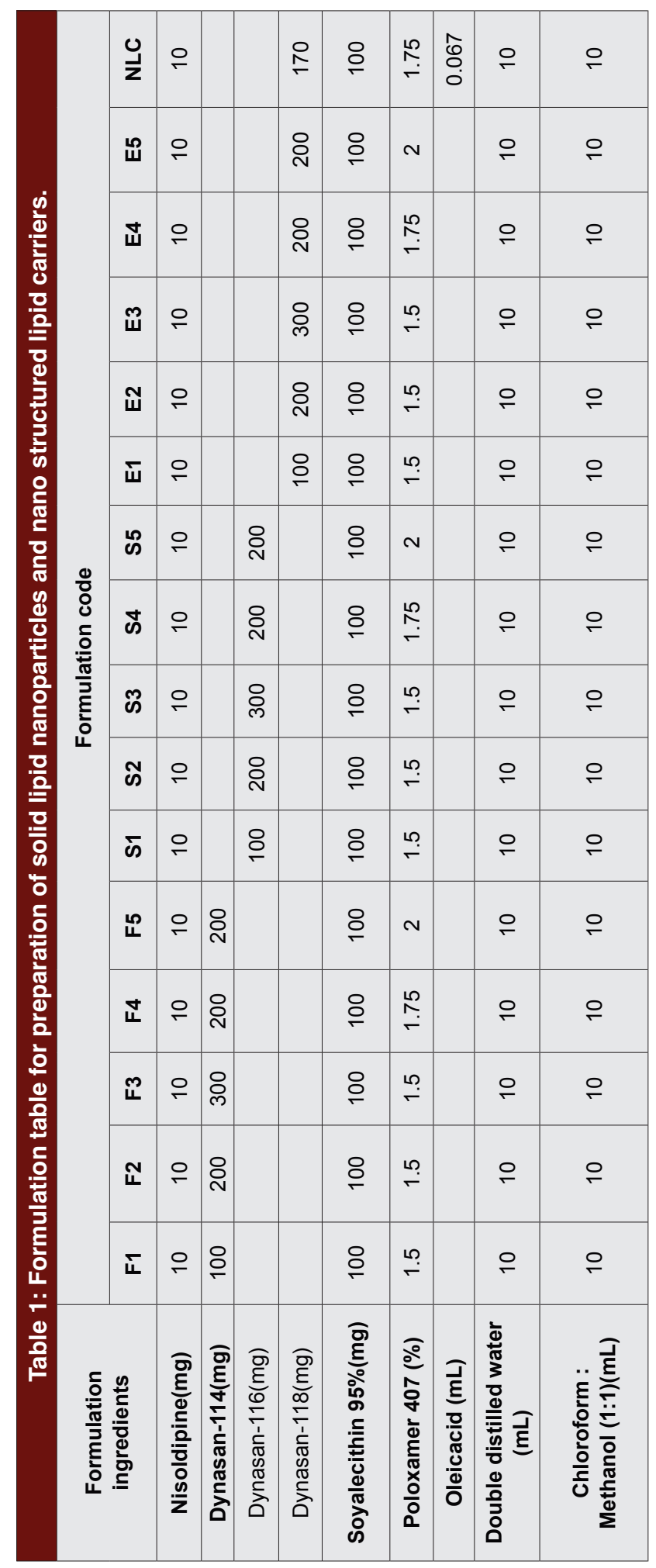

\section{Physicochemical properties}

The aqueous dispersion of NSPSLN and NSPNLCs were light yellowish in color, odorless and fluid in nature. Even after centrifugation dispersion was stable and didn't show sedimentation. Hydrogel incorporated with aqueous dispersions of NSPSLN and NSPNLCs were light yellow in color and odorless with smooth appearance. The gels $\mathrm{pH}$ was at 6.5 .

\section{Particle size analysis}

After preparation, the aqueous dispersions were stored at room temperature and their particle size and polydispersity index was measured using Photon correlation spectroscopy. After particle size analysis of the all formulations made with three solid lipids, results are summarized in Figure 1 and Table 2. The particle sizes of all formulations ranged from $160.23 \mathrm{~nm}$ to $330.25 \mathrm{~nm}$, PDI from 0.20 to 0.44 . It could also be observed that the particle size increased with the increase of lipid concentration and also PDI value decreased with the increase of lipid concentration. Based on the above results, selecting the optimizing formulation variables like lipid and surfactant concentrations were $200 \mathrm{mg}$ and $1.75 \%$ respectively. Optimizing process variables like homogenization time and ultrasonication time were $10 \mathrm{~min}$ and $20 \mathrm{~min}$. Table 3 shows the photon correlation spectroscopy (PCS) mean particle size and polydispersity index after 1, 30 and 60 days of storage at room and refrigerator temperatures. It could also observe that increment of particle size and PDI value are higher when formulations are stored at room temperature compared to stored in refrigerator.

\section{Zeta Potential (ל)}

Zetapotential measurement allows prediction about stability during the storage of colloidal dispersions. In general, aggregation of particles is less likely to occur for charge particles. For lipid nanoparticles the surface having the charge is negative. The zetapotential determination of aqueous of SLN and NLCs were performed during storage at room temperature and refrigerator conditions. The zeta potential and standard deviation values of all formulations are summarized in Figure 2 and Table 2. Agreement of these results with theory says that increased zetapotential values means that increased stability by electro repulsion. Increased zetapotential values were observed when increment in the concentration of lipid and surfactant. The zetapotential values of optimized E4 and E4 NLCs measured on $1^{\text {st }}$ day, after 30 days and after 60days of storage at room and refrigerator conditions, reveals that a slight decrease in the zetapotential of the lipid nanoparticles 
during storage time at both temperature. In comparison to E4 (NSP SLN with Dynasan118) for with the same lipid content E4 NLCs formulation shows lower zetapotential (Table 3).

\section{Drug content and Entrapment efficiency}

Drug content results showed that concentration of Nisoldipine in the total system ranged from $9 \mathrm{mg}$ to 9.6mg for different SLN/NLCs formulations. The percentage of drug which is incorporated in the lipid matrix (entrapment efficiency) was evaluated on day 1 for all formulations. Entrapment efficiency of all formulations ranged from 72 to $97 \%$ on day 1 . The results are shown in Figure 3 and Table 2. Incorporation of Nisoldipine led to high entrapment efficiency, probably because of their lipophilic character. The sample with lower lipid concentration shows lower entrapment efficiency but in case of surfactant, higher concentra-

Table 2: Particle size, PDI and zetapotential of Nisoldipine in SLN and NLCs formulations (mean \pm SD).

\begin{tabular}{|c|c|c|c|c|c|}
\hline $\begin{array}{l}\text { Formulation } \\
\text { code }\end{array}$ & $\begin{array}{c}\text { Size } \\
(\mathrm{nm}) \pm \mathrm{SD}\end{array}$ & PDI $\pm S D$ & $\begin{array}{l}\text { Zeta potential } \\
(\mathrm{mV}) \pm S D\end{array}$ & $\begin{array}{l}\text { Total drug content } \\
\text { (mg) }\end{array}$ & $\begin{array}{c}\text { Entrapment effficiency } \\
(\%)\end{array}$ \\
\hline F1 & $160.23 \pm 5.96$ & $0.44 \pm 0.028$ & $15.9 \pm 1.87$ & $9.09 \pm 0.05$ & $72.47 \pm 0.21$ \\
\hline F2 & $172.52 \pm 7.52$ & $0.37 \pm 0.035$ & $18.7 \pm 1.78$ & $9.13 \pm 0.09$ & $75.82 \pm 0.11$ \\
\hline F3 & $230.95 \pm 3.45$ & $0.36 \pm 0.013$ & $20.2 \pm 1.01$ & $9.37 \pm 0.02$ & $82.71 \pm 0.31$ \\
\hline F4 & $167.80 \pm 5.87$ & $0.25 \pm 0.016$ & $21.9 \pm 1.87$ & $9.52 \pm 0.01$ & $89.23 \pm 0.10$ \\
\hline F5 & $192.23 \pm 2.89$ & $0.39 \pm 0.012$ & $21.7 \pm 1.78$ & $9.16 \pm 0.07$ & $86.08 \pm 0.24$ \\
\hline s1 & $200.42 \pm 3.12$ & $0.35 \pm 0.018$ & $18.2 \pm 1.34$ & $9.02 \pm 0.04$ & $84.35 \pm 0.42$ \\
\hline S2 & $260.52 \pm 4.25$ & $0.31 \pm 0.021$ & $23.4 \pm 1.31$ & $9.23 \pm 0.01$ & $87.58 \pm 0.21$ \\
\hline S3 & $318.28 \pm 6.28$ & $0.27 \pm 0.017$ & $20.1 \pm 1.72$ & $9.47 \pm 0.02$ & $89.47 \pm 0.31$ \\
\hline S4 & $245.30 \pm 4.92$ & $0.21 \pm 0.031$ & $25.9 \pm 1.56$ & $9.54 \pm 0.07$ & $91.38 \pm 0.21$ \\
\hline S5 & $271.76 \pm 8.52$ & $0.28 \pm 0.084$ & $23.7 \pm 1.45$ & $9.40 \pm 0.03$ & $89.26 \pm 0.25$ \\
\hline E1 & $220.23 \pm 5.26$ & $0.26 \pm 0.028$ & $22.1 \pm 1.58$ & $9.03 \pm 0.05$ & $86.36 \pm 0.32$ \\
\hline E2 & $290.52 \pm 2.52$ & $0.21 \pm 0.035$ & $25.3 \pm 1.25$ & $9.27 \pm 0.03$ & $90.28 \pm 0.41$ \\
\hline E3 & $330.25 \pm 3.45$ & $0.27 \pm 0.013$ & $23.2 \pm 1.01$ & $9.39 \pm 0.08$ & $91.75 \pm 0.25$ \\
\hline E4 & $260.73 \pm 4.47$ & $0.20 \pm 0.097$ & $29.1 \pm 2.16$ & $9.60 \pm 0.02$ & $93.56 \pm 0.30$ \\
\hline E5 & $300.29 \pm 5.79$ & $0.23 \pm 0.015$ & $26.3 \pm 2.06$ & $9.23 \pm 0.01$ & $90.06 \pm 0.09$ \\
\hline NLC & $140.79 \pm 12.9$ & $0.29 \pm 0.013$ & $27.9 \pm 1.97$ & $9.66 \pm 0.07$ & $97.07 \pm 0.07$ \\
\hline
\end{tabular}

\begin{tabular}{|c|c|c|c|c|c|c|c|}
\hline \multirow{2}{*}{ Formulation Code } & \multirow{2}{*}{ Day } & \multicolumn{3}{|c|}{ At room temperature } & \multicolumn{3}{|c|}{ At refrigerated temperature } \\
\hline & & Size(nm) & PDI & Zeta potential(mV) & $\operatorname{Size}(\mathrm{nm})$ & PDI & Zeta potential(mV) \\
\hline \multirow{3}{*}{ E4 } & 1 & $260.73 \pm 4.47$ & $0.20 \pm 0.09$ & $-29.1 \pm 2.16$ & $260.73 \pm 4.47$ & $0.20 \pm 0.09$ & $-29.1 \pm 2.16$ \\
\hline & 30 & $285.56 \pm 8.58$ & $0.28 \pm 0.14$ & $-25.3 \pm 2.06$ & $272.23 \pm 2.89$ & $0.31 \pm 0.01$ & $-26.1 \pm 2.04$ \\
\hline & 60 & $320.9 \pm 11.49$ & $0.47 \pm 0.06$ & $-22.4 \pm 2.08$ & $291.01 \pm 8.54$ & $0.34 \pm 0.03$ & $-24.6 \pm 2.12$ \\
\hline \multirow{3}{*}{ E4 NLC } & 1 & $140.79 \pm 12.9$ & $0.29 \pm 0.01$ & $-27.9 \pm 1.97$ & $140.79 \pm 12.9$ & $0.29 \pm 0.01$ & $-27.9 \pm 1.97$ \\
\hline & 30 & $152.64 \pm 9.34$ & $0.37 \pm 0.03$ & $-23.3 \pm 1.67$ & $147.09 \pm 3.14$ & $0.32 \pm 0.01$ & $-25.4 \pm 1.26$ \\
\hline & 60 & $165.28 \pm 7.36$ & $0.44 \pm 0.05$ & $-20.8 \pm 1.80$ & $152.29 \pm 4.32$ & $0.38 \pm 0.04$ & $-22.1 \pm 1.32$ \\
\hline
\end{tabular}


tion shows lower entrapment efficiency, for all SLN formulations. Transition of dispersed lipid from meta stable form to stable form might occur slowly on storage because of small particle size and the presence of surfactants may lead to drug expulsion of from SLN formulations. ${ }^{17-19}$ E4 NSP NLCs are responsible for higher entrapment efficiency in comparison to all SLN formulations. This result due to the binary mixture of liquid and solid lipids, resulting in only a very weak crystallization. ${ }^{20,21}$ For all the formulations, the entrapment efficiency was higher than $80 \%$.

\section{Drug-Excipients compatibility studies (DSC Analysis)}

A differential Scanning Calorimetry (METTTLER) was used to study the thermal analysis of drug-excipients compatibility (Figure 4). Pure drug (Nisoldipine), freeze dried SLN preparations of three lipids Dynasan 114, 116 and 118 products were scanned in the temperature range of $50-250^{\circ} \mathrm{C}$. Analysis performed under a Nitrogen purge. Nisoldipine showed a sharp endothermic peak at $157.34^{\circ} \mathrm{C}$. Lyophilized product of Nisoldipine loaded Dynasan 114,116 and 118 SLN showed sharp endothermic peaks at $162.36^{\circ} \mathrm{C}, 163.46^{\circ} \mathrm{C}$ and $163.45^{\circ} \mathrm{C}$ respectively but intensity of the peak was decreased. This characteristic peak intensity was not observed in nisoldipine loaded SLN. The absence of detectable crystalline domains of nisoldipine in drug-loaded SLNs clearly indicates that nisoldipine encapsulated in SLNs is in the amorphous or disordered-crystalline phase or in the solid-state solubilized form in the polymer matrix. The endothermic peaks of Dynasan-114,116 and 118 were found approximately at $\sim 61.51^{\circ} \mathrm{C}, \sim 68.72^{\circ} \mathrm{C}$ and $\sim 74.55^{\circ} \mathrm{C}$ respectively due to glass transition temperature ( $\mathrm{Tg}$ ) of Polymer. Thus the observation indicated that there was no interaction between Nisoldipine and three lipids.

\section{Transmission Electron Microscopy}

The image of TEM of the NSPSLN and NSPNLC was shown in Figure $5 \mathrm{~A}$ and $\mathrm{B}$, respectively. The result shows that the particles diameters vary from about 150 to 300 $\mathrm{nm}$. There is no significant difference in the diameter between SLN and NLCs.

\section{Drug release from SLN and NLC by dialysis method}

From above results formulations containing Dynasan-114, 116 and 118 (F4, S4 and E4) were selected for in-vitro drug release studies. Results showed (Figure 6 and 7) maximum drug release of $65.54 \%, 71.16 \%$ and $75.96 \%$ respectively in $\mathrm{pH} 7.4$ phosphate buffer with $0.75 \%$ SLS. The drug release pattern form formulation of SLNs F4, S4 and E4 showed biphasic release behavior consisting of initial burst release of $20 \%$ to $30 \%$ within one hour, followed by sustained drug release about 60 to $75 \%$ at the end of $24^{\text {th }} \mathrm{hr}$. Presence of adsorbed drug on the surface of solid lipid nanoparticles is the reason for initial burst drug release and increased diffusional path length and hindering effect of surrounding solid lipid shell are the reasons for further sustained drug release. The SLNs with Dynasan 114, 116 and 118 incorporated into carbopol 934p gels (F4 gel, S4 gel and E4 gel) showed release of $29.94 \%, 24.68 \%$ and $37.93 \%$ respectively. E4 formulation showed highest percentage drug release among all the prepared SLNs, for that E4 formulation selected for further studies. NLC formulation (Dynasan 118) and gel loaded with NLCs of Dynasan 118 showed maximum release of $82.09 \%$ and $40.76 \%$ respectively. Further, all SLNs released drug relatively slowly when compared to that of NLCs. For preparation of NLCs $30 \%$ of solid lipid is replaced with liquid lipid (Oleic acid) due to this reason NLCs showing higher drug release compared to SLNs.

\section{Release Kinetics}

To know the release kinetics drug release data can be fit into different drug release kinetics results shown in Table 4. Release of drug from all the formulations followed zero order and higuchi, the best fit with the highest correlation coefficients were shown in zero order followed by higuchi plot. The mechanism of release is by diffusion as indicated by $R^{2}$ value of higuchi and $n$ value of korsemeyer peppas equation. The percentage drug release was proportional to square root of time (Higuchi model) indicating that drug release from SLN and NLC and gels enriched with SLN/NLC is diffusion controlled. More over the plots of log percentage released vs. log time showed a high level linearity as given in Table 4, which in turn another conformation that the release is diffusion controlled.

\section{Ex vivo permeation studies}

The ex vivo skin permeation of Nisoldipine loaded SLN formulations (F4, S4 and E4), NLC (E4 NLC), gels enriched with F4, S4, E4, NLC and drug solution were showed in Figure 8 and 9. NSPNLC exhibited the greatest $(68.10 \%)$ percentage release of drug permeation in $24 \mathrm{hr}$. the amount of drug permeated per square centimeter of the effective diffusional area through the rat abdominal skin when plotted against time, the permeation profiles of drug seem to follow Higuchi's equation $\left(R^{2}=0.978\right.$ to 0.991$)$ and zero order kinetics as it is evidenced by correlation coefficients (0.791 to 0.977 ). The flux for every formulation was shown in Figure 


\begin{tabular}{|} 
Table 4: Regression coefficient (R2) values of Nisoldipine loaded SLN and NLC formulations through dialysis \\
membrane. \\
\hline Formulations & Zero order & First order & Higuchi & Korsmeyer-peppas & $n$ value \\
\hline F4 & 0.983 & 0.943 & 0.988 & 0.956 & 0.38 \\
\hline S4 & 0.943 & 0.874 & 0.96 & 0.978 & 0.345 \\
\hline E4 & 0.936 & 0.889 & 0.963 & 0.961 & 0.328 \\
\hline F4 gel & 0.874 & 0.708 & 0.834 & 0.946 & 0.437 \\
\hline S4 gel & 0.902 & 0.741 & 0.846 & 0.934 & 0.365 \\
\hline E4 gel & 0.942 & 0.828 & 0.931 & 0.98 & 0.511 \\
\hline NLC & 0.912 & 0.856 & 0.945 & 0.963 & 0.323 \\
\hline NLC gel & 0.944 & 0.893 & 0.969 & 0.978 & 0.291 \\
\hline
\end{tabular}

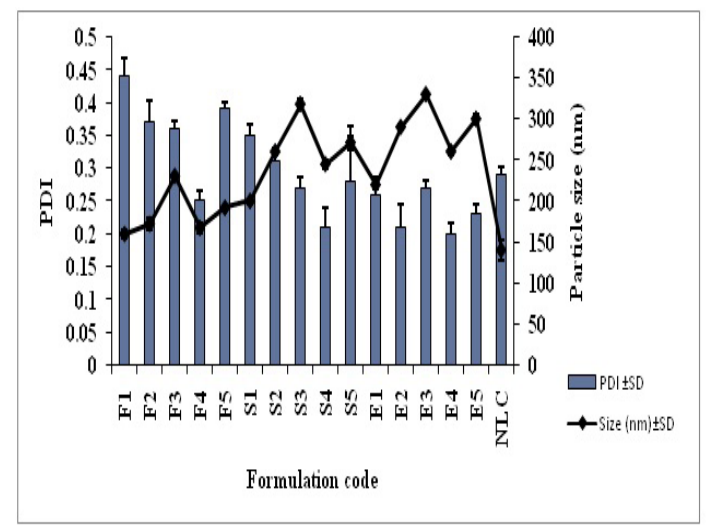

Figure 1: Particles size and PDI of all SLN and NLCs formulations.

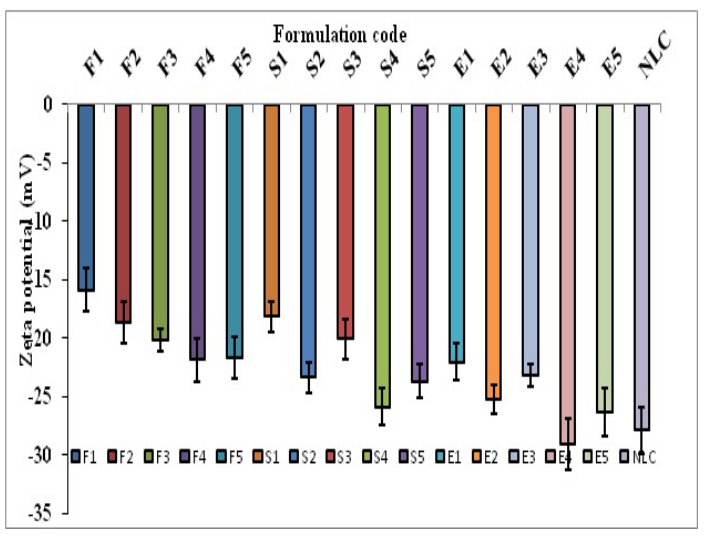

Figure 2: Zeta potential values of all SLN and NLCs formulations.

10. E4 SLN and E4 NLCs showed higher flux 0.019 $\mathrm{mg} / \mathrm{cm}^{2} / \mathrm{hr}$ and $0.018 \mathrm{mg} / \mathrm{cm}^{2} / \mathrm{hr}$. the results of $\mathrm{drug}$ release from NSPSLN, NSPNLC and gels enriched with SLN/NLC through the rat abdominal skin confirmed that Nisoldipine was released and permeated through the human skin. Finally percentage of drug permeated through rat kin was correlated against percentage of drug release by using in-vitro release test for opti-

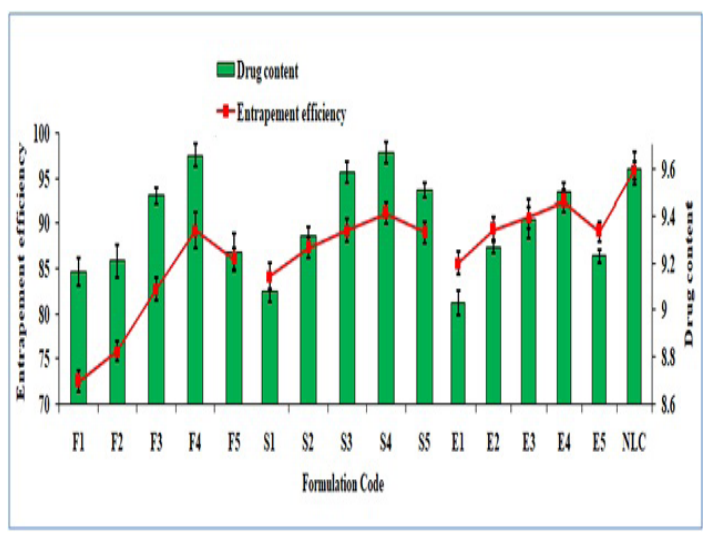

Figure 3: Drug content and entrapment efficiency of all formulations.

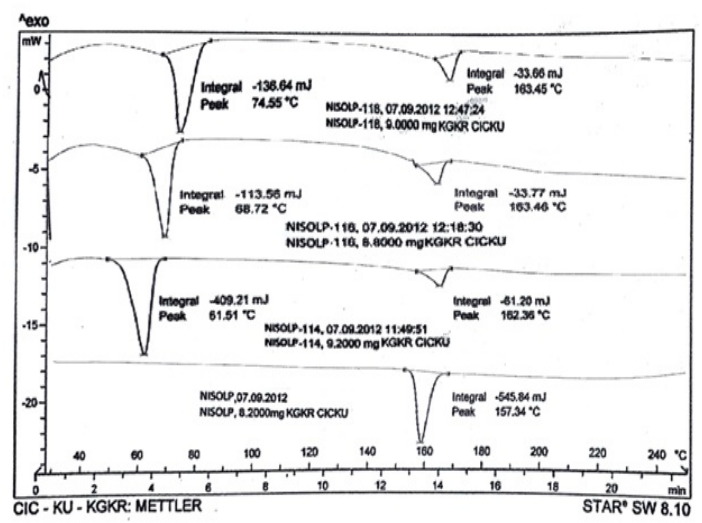

Figure 4: DSC studies of Nisoldipine and various formulations.

mized formulations, Figure 11 and 12 shows relationship between the percentage of Nisoldipine permeated in ex-vivo and percentage of Nisoldipine in in-vitro. The straight line and high correlation coefficient 0.979 and 0.993 of E4 NLCs and E4 NLCs gel respectively proved the good correlation between ex-vivo permeation and invitro drug release studies. Hence by the complete differences in the test conditions of ex-vivo and in-vitro release 


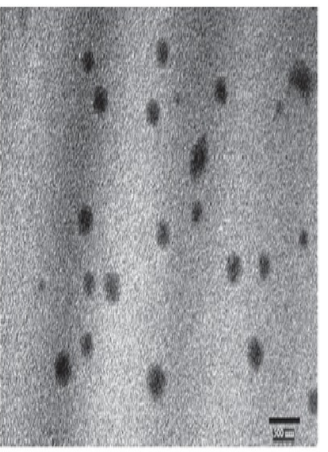

(A)

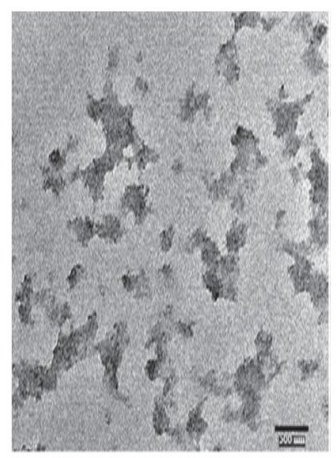

(B)
Figure 5: (A) Transmission electron microscope (TEM) image of nisoldipine solid lipid nanoparticles (NSPSLN). (B) TEM image of nisoldipine nanostructured lipid carrier (NSPNLC).

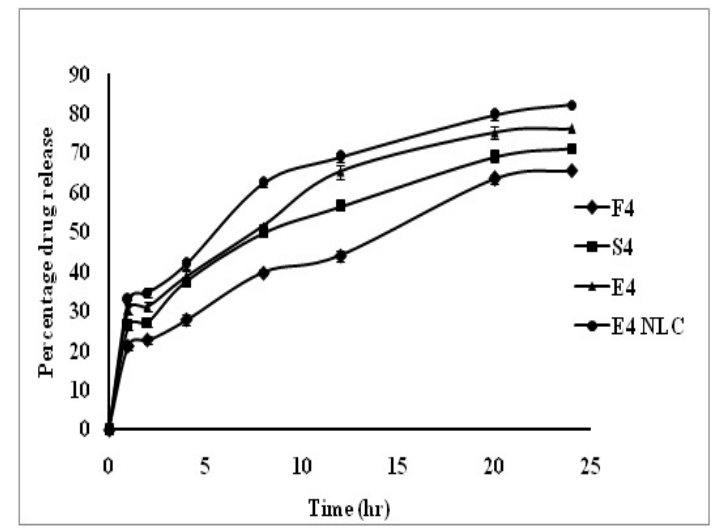

Figure 6: In vitro drug release studies of SLN/ NLCs formulations.

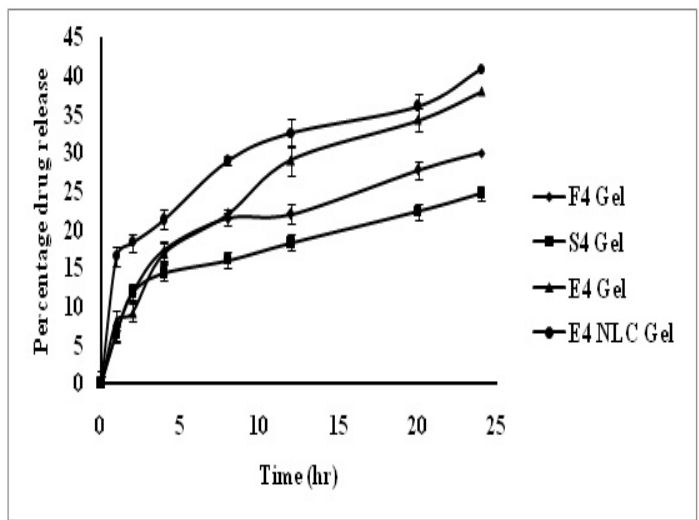

Figure 7: In vitro drug release studies of gels enriched with SLN/NLCs.

studies, the high correlation and coincidence of in-vitro and ex-vivo release profile, it can be concluded that such a transdermal gel systems could be a useful carrier in transdermal drug delivery systems.

\section{Physical stability studies}

Nisoldipine loaded SLN were stored at room and refrigerated temperature for 60 days and average size, zeta

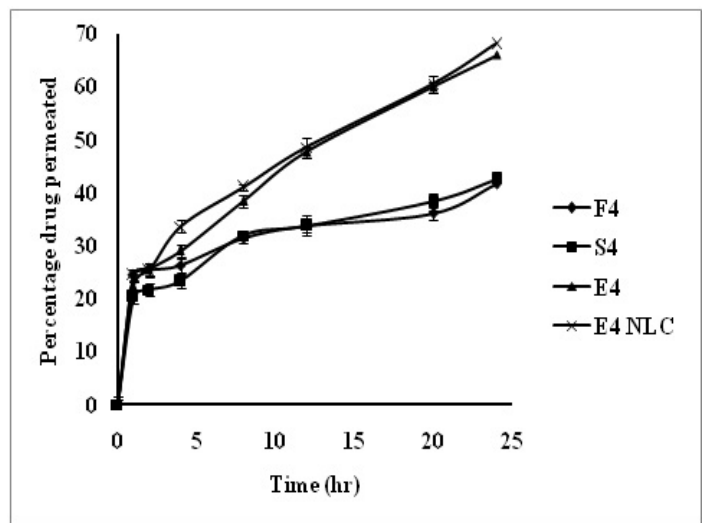

Figure 8: EX vivo permeation studies of SLN/NLCs formulations.

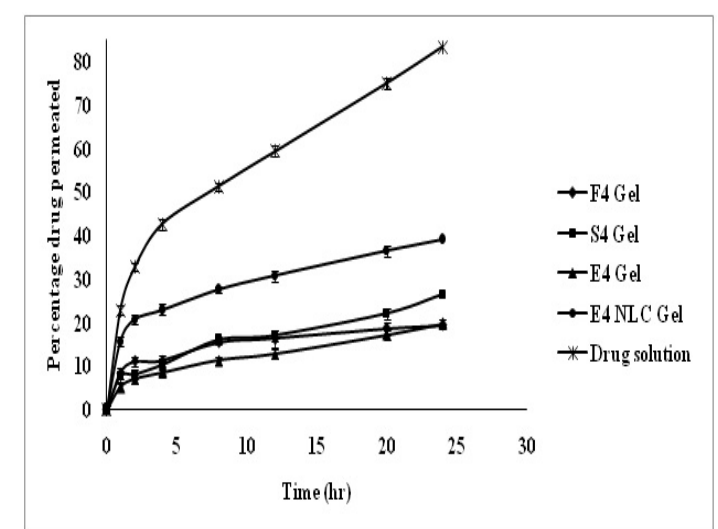

Figure 9: Ex vivo permeation studies of gel enriched with SLN/NLCs formulations and drug solution.

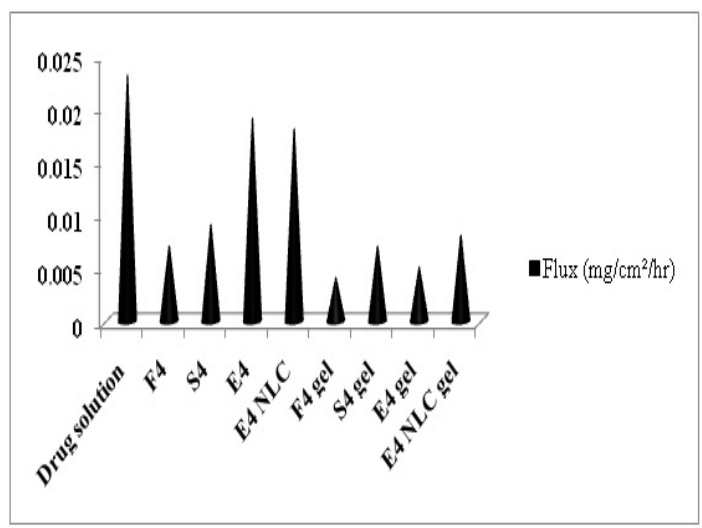

Figure 10: Calculation of flux of various SLN, NLCs formulation and drug solution.

potential and poly dispersity index (PDI) were determined. Stability studies were conducted for optimized formulation (E4) and NLC which showed better size, PDI, zeta potential, entrapment efficiency and release characteristics. Some changes were noted in size, PDI and zeta potential values, which indicated the susceptibility for stability problems during storage at room temperature and $4^{\circ} \mathrm{C}$. Hence, lyophilization technique 


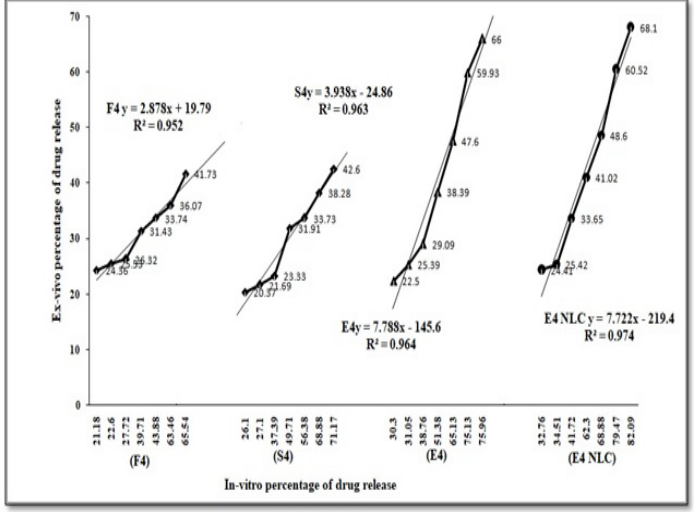

Figure 11: In vitro and ex vivo correlation between the cumulative $\%$ of drug released in vitro and \% of drug permeated ex vivo of optimized SLN and NLC.

Table 5: Characterization of optimized formulation E4 before and after lyophilization.

\begin{tabular}{|c|c|c|}
\hline Parameters & $\begin{array}{c}\text { Before } \\
\text { lyophilization }\end{array}$ & $\begin{array}{c}\text { After } \\
\text { lyophilization }\end{array}$ \\
\hline Size $(\mathrm{nm})$ & 260.73 & 623.3 \\
\hline PDI & 0.2 & 0.39 \\
\hline Zeta potential $(\mathrm{mV})$ & 29.1 & 31.2 \\
\hline
\end{tabular}

was used to overcome this problem. The optimized formulation (E4) was subjected to lyophilization. Mannitol $(10 \%)$ was used as cryoprotectant. Characterization of optimized formulation E4 before and after lyophilization results were tabulated in Table 5. Due to lyophilization the size of NLCs increased due to adhesion of smaller NLCs particles and PDI was also found to be increased.

\section{CONCLUSION}

In this work, efforts were made to prepare stable solid lipid nanoparticles and nanostructured lipid carriers for characterization of in-vitro and ex-vivo release of Nisoldipine from SLNs, NLCs and gels with SLN/NLC. Stability studies were conducted for finally optimized formulation (E4) at room temperature and $4^{\circ} \mathrm{C}$ for 2 months; some changes were noticed in size, PDI and zeta potential values, which indicated the susceptibility for stability problems during storage at room temperature and $4^{\circ} \mathrm{C}$. Hence, lyophilization technique was used to overcome this problem. NSPNLC dispersion showed faster release in comparison NSPSLN dispersion and gels enriched with SLN/NLCs. In vitro release of NSPNLC, NSPSLN dispersions and gels enriched with SLN/NLC followed Higuchi and zero order kinetics. Both SLN and NLC showed a sustained drug release over period of $24 \mathrm{hr}$ but the sustained effect was more pronounced with the SLN and NLC gel formulations. In

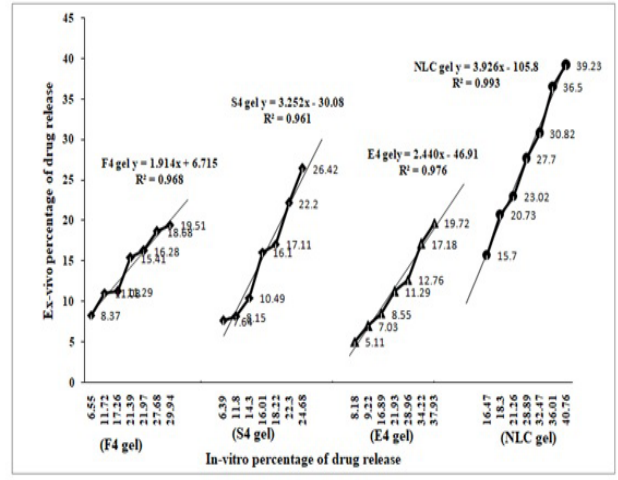

Figure 12: In vitro and ex vivo correlation between cumulative $\%$ of drug released in vitro and \% of drug permeated ex vivo of optimized SLN Gels and NLC Gels.

conclusion, the developed formulations are promising alternative drug carriers for transdermal drug delivery. Pharmacokinetic and pharmacodynamics evaluation of these systems in animals and humans is necessary to confirm these findings.

\section{ACKNOWLEDGEMENT}

Authors are thankful to Women Scientist-A, DST, Government of India for providing financial assistance in the form of Fellowship during the project. Project No. SR/WOS-A/LS-71/2017. Authors are also thankful to Aurabindo Pharma, Hyderabad for providing Nisoldipine as a gift sample.

\section{CONFLICT OF INTEREST}

The authors declare no conflict of interest.

\section{ABBREVIATIONS}

SLN: Solid Lipid Nanoparticles; NLCs: Nanostructured Lipid Carriers; NSP: Nisoldipine; NSPSLN: Nisoldipine solid lipid nanoparticles; NSPNLCs: Nisoldipine nanostructured lipid carriers; PDI: Poly Dispersity Index.

\section{REFERENCES}

1. Muller RH, Madar K, Gohal S. Solid Lipid Nanoparticles (SLN) for controlled drug delivery: A review of the state of art. Eur J Pharm Biopharm. 2001;50(1):161-77.

2. Muller RH, Mehnert W, Lucks JS, Schwarz C, Muhlen AZ, Weyhers H, et al. Solid lipid nanoparticles (SLN): An alternative colloidal carrier systems for cotrolled drug delivery. Eur J Pharm Biopharm. 1995;41:62-9.

3. Manjunath K, Reddy JS, Venkateswarlu V. Solid lipid nanoparticles as drug delivery systems. Methods Find Exp Clin Pharmacol. 2005;27(2):127-44.

4. Muller RH. Solid liquid (semi solid) lipid particles and method of producing highly concentrated lipid particle dispersions. German Patent Application. 1994;5:203. 
5. Siekmann B, Westensen K. Submicron sized parenteral carrier system based on solid lipids. Pharm Pharmacol. 1992;1:123-6.

6. Muller RH, Radtke M, Wissing SA. Solid lipid nanoparticles (SLN) and nanostructured lipid carriers (NLC) in cosmetic and dermatological preparations. Adv Drug Deliv Rev. 2002a;54:S31-55.

7. Muller RH, Radtke M, Wissing SA. Nanostructured lipid matrices for improved mmicroencapsulation of drugs. Int J Pharm. 2002b;242(1-2):121-8.

8. Muller RH, Ruhl D, Runge S. Biodegradation of solid lipid nanoparticles as a function of lipase incubation time. Int J Pharm. 1996b;144(1):115-21.

9. Wissing SA, Muller RH. Cosmetic applications for solid lipid nanoparticles (SLN). Int J Pharm. 2003;254(1):65-8.

10. Ramesh G, Madhusudan RY. Formulation optimization and evaluation of microemulsion based transdermal therapeutic system for nitrendipine. J Dis Sci Tech. 2012;33(02):223-33.

11. Bhaskar K, Krishna MC, Lingam M, Venkateswarlu V, Madhusudan RY, Ravichandran V. Development of SLN and NLC enriched hydrogels for transdermal delivery of Nitrendiine: In-vitro, ex-vivo and in-vivo Charcteristics. Drug Del Ind Pharm. 2008;35(1):98-113.

12. Venkateswarlu V, Manjunath K. Preparation, characterization and in-vitro release kinetics of clozapine solid lipid nanoparticles. J Control Release. 2004;5(3):627-38.

13. Pavan KP, Gayatri P, Reddy S, Jaganmohan S, Madhusudan RY. Atorvastatin loaded solid lipid nanoparticles: Formulation, optimization and in-vitro Charcterization. IOSR Journal of Pharmacy. 2012;2(5):23-32.
14. Gayatri P, Ajitha M, Pavan KP, Madhusudan RY. Development and characterization of Nisoldipine matrix type Transdermal films in-vitro and exvivo evaluation American Sientific Res. J for Eng Tech and Scis. 2016;23(1):114.

15. Dhonge KD, Patil DM, Phatal AA, Pachpute DS. Formulation development and characterization of transdermal film of Nisoldipine. Pharma Science Monitor. 2014;5(3):S1.

16. Suwanpiodokkul N, Thongnopnua P, Umprayan K. Transdermal delivery of Zidovudine (AZT): The effects of vehicles, enhancers and polymer membranes on permeation across cadaver pig skin. AAPS Pharm Sci Tech. 2004;5(3):1-7

17. Westen K, Siekmann B, Koch M. Investigations on the physical state of the lipid nanoparticles by synchrotron radiation X-ray diffraction. Int J Pharm. 1993;93(1-3):189-99.

18. Bunjes $\mathrm{H}$, Westesen $\mathrm{K}$. Do nanoparticles prepared from lipids solids at room temperature always possess a solid lipid matrix?. Int $\mathrm{J}$ Pharm. 1995;115(1):129-31.

19. Mehnert W, Mader K. Solid lipid nanoparticles production, characterization and applications. Adv Drug Delivery Review. 2001;47:165-96.

20. Jenning V, Mader K, Gohla SH. Solid lipid nanoparticles based on binary mixtures of liquid and solid lipids: A ${ }^{1}$ H-NMR study. Int J Pharm. 2000;205(12):15-21.

21. Jenning V, Thunemann AF, Gohla SH. Charcterization of a novel solid lipid nanoparticles carrier system based on binary mixture of liquid and solid lipids. Int J Pharm. 2000;199(2):167-77.

\section{PICTORIAL ABSTRACT}
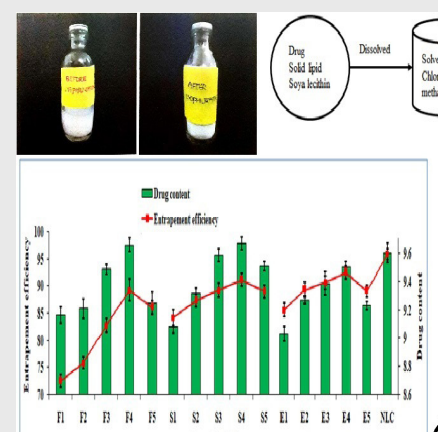

Fraulthin idide

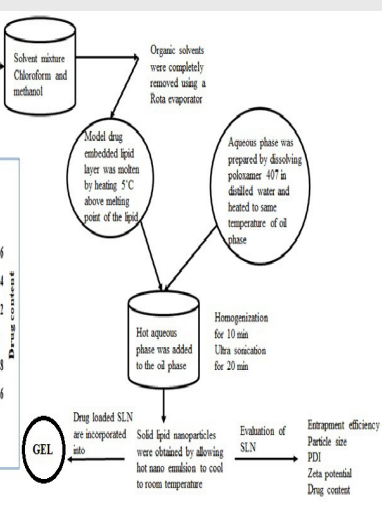

- Nisoldipine loaded SLN and NLCs were prepared by using different solid lipids and liquid lipids for application to transdermal drug delivery.

- Both SLN and NLC showed a sustained drug release over period of $24 \mathrm{hr}$ but the sustained effect was more pronounced with the SLN and NLC gel formulations.

\section{About Authors}

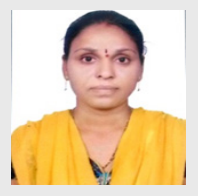

Suddala Shirisha: PI, Women Scientist-A, Research scholar in University Department of Pharmaceutical Sciences, Utkal University, Vanivihar. Bhubaneswar, Orissa-75100.

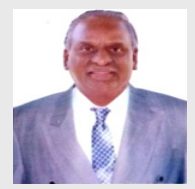

Prof. (Dr.). Yamsani Madhusudan Rao: Director, Vaagdevi Group of colleges, Warangal (T.S), India. He has five patents to his credit and has also authored several publications which include eight books related to Pharmaceutical Technology sector. He is guiding several M.Pharm and $\mathrm{Ph} . \mathrm{D}$ candidates for research projects sponsored by various government agencies. He has delivered several invited lecturers.

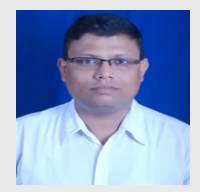

Dr. Sunit Kumar Sahoo: Lecturer in pharmaceutics, University Department of Pharmaceutical Sciences, Utkal University, Vanivihar, Bhubaneswar, Orissa-75100. With teaching experience of academic thirteen years and research twelve years, he has to his title fourty eight publications in various national and international journals. He has participated/ presented 65 national and international conferences/ seminars/ workshops.

Cite this article: Shirisha S, Saraswathi A, Sahoo SK, Rao YM. Formulation and Evaluation of Nisoldipine Loaded Solid Lipid Nanoparticles and Nanostructured Lipid Carriers: Application to Transdermal Delivery. Indian J of Pharmaceutical Education and Research. 2020;54(2s):s117-s127. 